\title{
INTERCÂMBIO
}

\section{MM: CONTRIBUIÇÃO AO DESENVOLVIMENTO DAS RELAÇÕES BRASIL- ALEMANHA NO CAMPO DA NEUROCIRURGIA}

\author{
FELICIANO PINTO*, LILIAN P. MACHADO**
}

Em 1980 foi idealizado o primeiro Seminário de Pós-Graduação para jovens neurocirurgiões brasileiros a que se denominou MM (Seminário sobre Modernos Métodos em Neurocirurgia), no Serviço de Neurocirurgia do KJinikum Steglitz da Universidade Livre de Berlim, com duração de quatro semanas. O objetivo desse Seminário era proporcionar a esses profissionais a oportunidade de viajar para a Europa e tomar contato com uma clínica neurocicúrgica moderna, observando o seu funcionamento e familirizando-se com os procedimentos recentemente introduzidos na especialidade.

Em 1981, o primeiro grupo (MM-1) viajou para Berlim. Vinte neurocirurgiões oriundos dos mais diversos pontos de nosso país, foram selecionados. Era de grande interesse que houvesse representatividade das diferentes regiões, guardadas as proporções da densidade populacional de neurocirurgiões das referidas cidades. A idéia do Prof. Mário Brock obteve o apoio do governo de Berlim e da Gari Duisberg Gesel Ischaft (Figura 1).

A organização do MM obedeceu desde o início, aos seguintes critérios de seleção: 1. idade limite de 30 anos (posteriormente ampliado para 40); 2. certificado de conclusão de treinamento em Neurocirurgia, em serviço credenciado pelas sociedades especializadas; 3 . vínculo com uma Universidade; 4. conhecimento de um idioma estrangeiro, de preferência alemão ou inglês; 5. nunca ter estado na Alemanha; 6. comprometer-se a viajar desacompanhado; 7. aceitaras normas disciplinares estabelecidas para todo o grupo.

Os candidatos do MM-1 foram designados pelos respectivos chefes de Serviço, tendo sido admitidos porém, três candidatos como ouvintes. A partir do MM-2 (1983), novos critérios de seleção e escolha foram aplicados. Os interessados foram orientados no sentido de remeter para o Tutor o currículo completo com os respectivos comprovantes. Também no MM-2, por sugestão do governo de Berlim, foram admitidos seis neurocirurgiões de outros países latino-americanos, a saber: dois equatorianos, dois argentinos e dois peruanos (Figuras 2 a 6 ).

O MM oferece aos interessados as seguintes vantagens: a. passagem aérea, classe turística, point-to-point, Rio de Janeiro-Berltm-Rio; b. alojamento no KJinikum, em quartos duplos; c. permissão para fazer as refeições no restaurante da Universidade; d. programa científico e social.

O esquema de trabalho científico inclui atividades práticas e teóricas. Para as atividades práticas, são organizados quatro grupos, a saber: 1. atividade no laboratorio de microcirurgia experimental; 2. treinamento cirúrgico (incluindo anatomia cirúrgica) e técnicas neurocirúrgicas no Departamento de Anatomia Patológica; 3. atividade no Centro Cirúrgico; 4. radiologia convencional, tomografia computadorizada e ressonância nuclear magnética. Essas atividades são realizadas das 8:30h às 12:30h. Após uma semana tem lugar uma rotação, de modo que, ao cabo de um mês cada grupo passou por todos os quatro setores. Das 13:30h às 15:00h, os mesmos grupos são instruídos em Sessões de: 1. Vídeo-tape; 2. Doppler; 3. Terapia Intensiva; 4. Neuropatologia. Entre 15:00h e 17:00h têm lugar conferências teóricas. Nas Figuras 7,8 e 9 mostram-se o organograma das atividades do MM-6.

Desde o MM-1 o Prof. Mário Brock demonstrou preocupação no sentido de proporcionar o conhecimento de inovações na Neurocirurgia e, já em 1981, o primeiro grupo tinha contato com novas técnicas, como: tomografia computadorizada do encéfalo; cisternoscopia do ângulo ponto cerebelar; estabilização da coluna vertebral com endoprótese; uso do aspirador ultrassônico em Neurocirurgia; craniotomia fronto-lateral na cirurgia dos aneurismas

Sociedade Mário Brock de Neurocirurgia (SMBN): *Secretário Geral; ** Secretária Auxiliar.

Dr. Feliciano Pinto - SMBN, Sociedade Mário Brock de Neurocirurgia - Av. Princesa Isabel 150 Grupo 604 22110-010 Rio de Janeiro RJ - Brasil. Fax (021) 275.0095. 


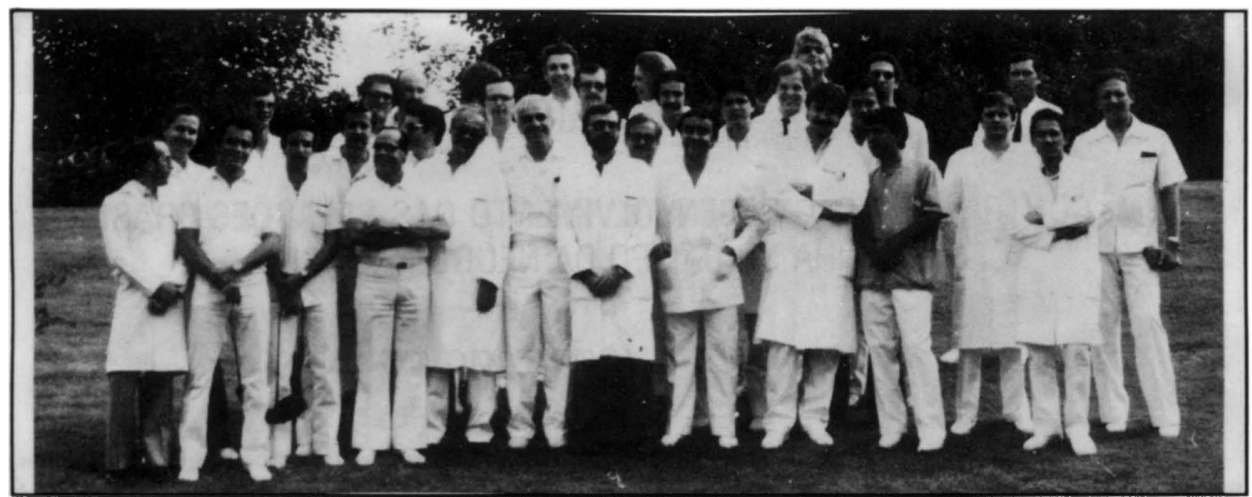

Figura 1. Integrantes do MM-1 (1981).

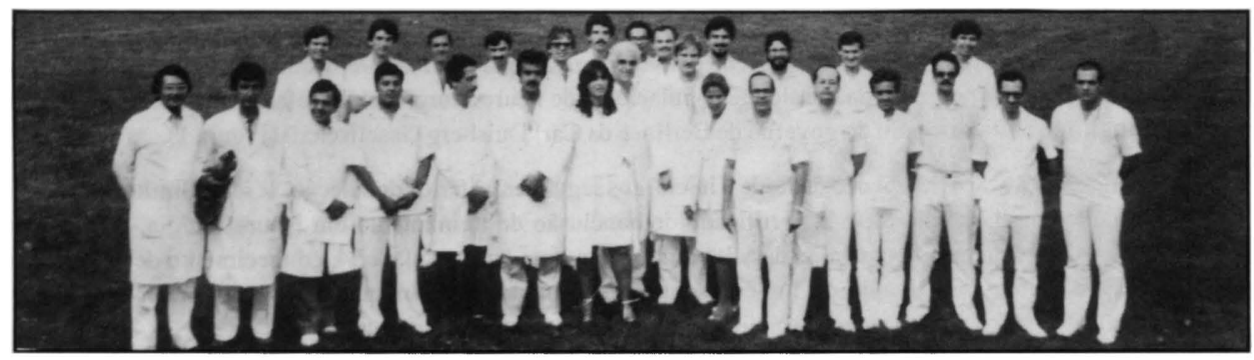

Figura 2. Integrantes do MM-2 (1983).

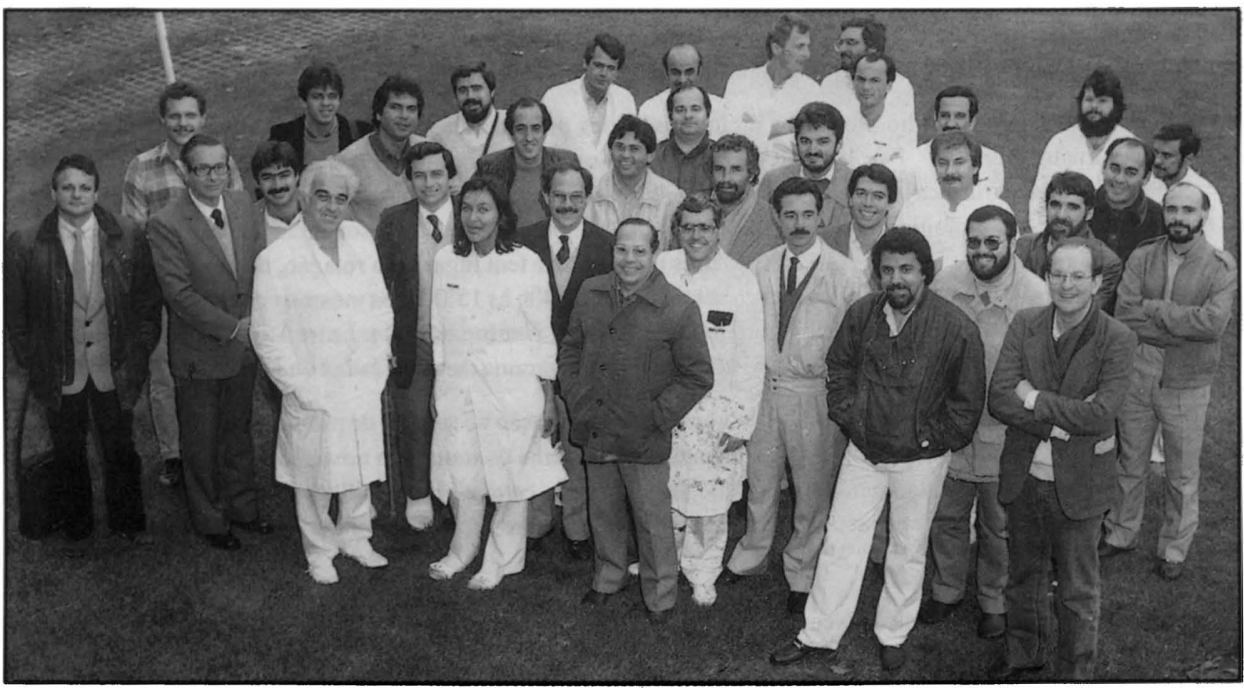

Figura 3. Integrantes do MM-3 (1985). 


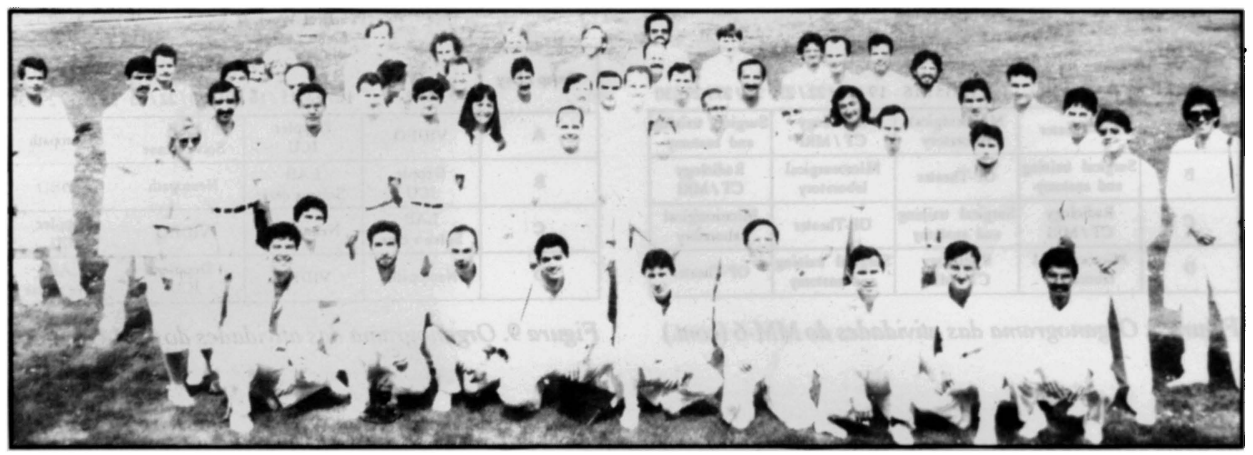

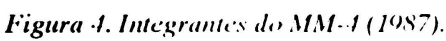

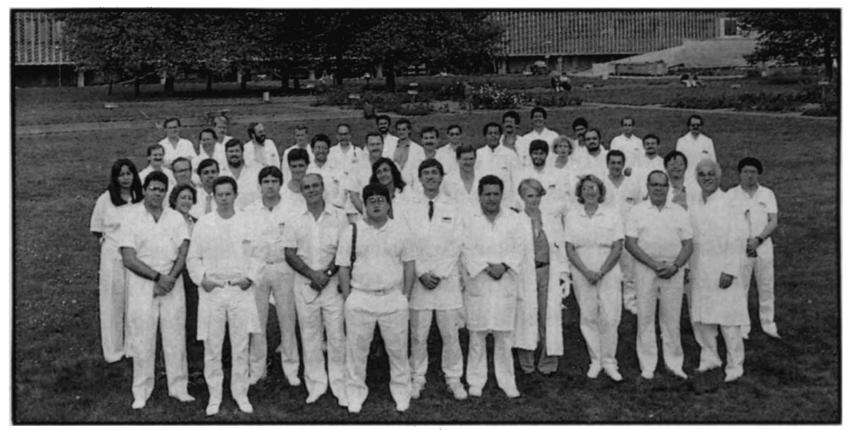

Fïgura 5. Integrantes do MM-.5 (1989).

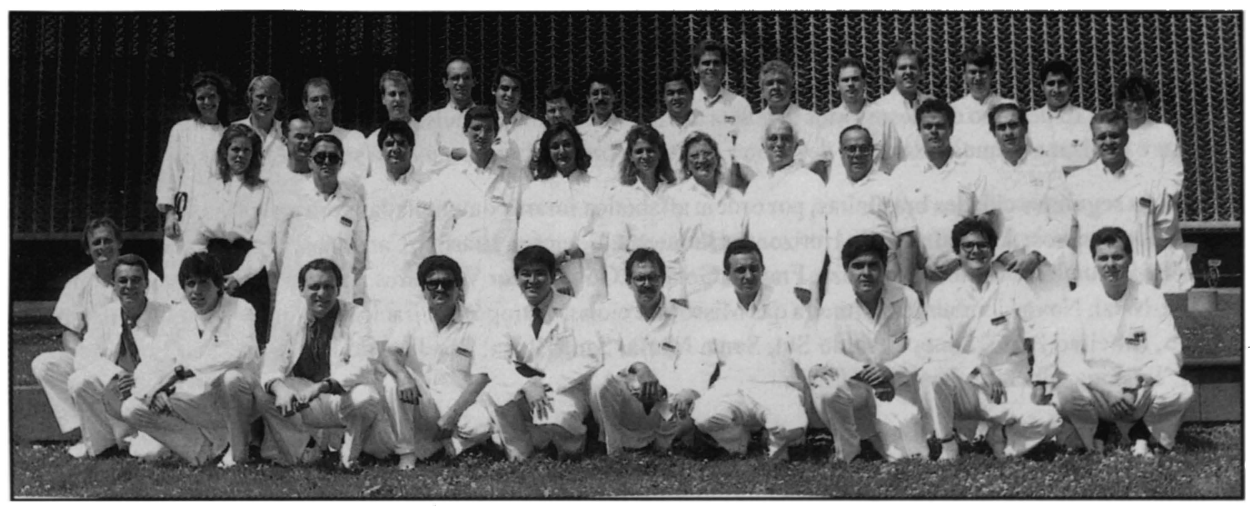

Figura 6. Integrantes do MM-6 (1993).

TIME TABLE

\begin{tabular}{|c|c|c|c|c|}
\hline & $12:$ & & $3: 30$ & \\
\hline Mon & Practical Work I & L & Practical Work II & Lectures \\
\hline Tue & Practical Work I & $\mathbf{U}$ & Practical Work II & Lectures \\
\hline \multicolumn{2}{|l|}{ Wed } & $\mathbf{N}$ & & \\
\hline Thu & Practical Work I & \multirow{2}{*}{$\begin{array}{l}\mathrm{C} \\
\mathrm{H}\end{array}$} & Practical Work II & Lectures \\
\hline Fri & Practical Work I & & Practical Work II & Lectures \\
\hline
\end{tabular}

Figura 7. Organograma das at:vidades do MM-6. 


\begin{tabular}{|c|c|c|c|c|}
\hline \multirow{3}{*}{ Groep / Day } & \multicolumn{3}{|c|}{$\begin{array}{c}\text { Practical Work I } \\
8: 30 \text { - 12:30 }\end{array}$} & \multirow[b]{3}{*}{$26 / 27 / 29 / 30$} \\
\hline & \multicolumn{3}{|c|}{ Juby } & \\
\hline & $5 / 6 / 8 / 9$ & $12 / 13 / 15 / 16$ & $19 / 20 / 22 / 23$ & \\
\hline $\mathbf{A}$ & OP-Theater & $\begin{array}{l}\text { Microsurgical } \\
\text { Labontory }\end{array}$ & $\begin{array}{l}\text { Rediology } \\
\text { CT / MRI }\end{array}$ & $\begin{array}{l}\text { Surgical training } \\
\text { and anatomy }\end{array}$ \\
\hline B & $\begin{array}{c}\text { Surgical training } \\
\text { snd anatomy }\end{array}$ & OP-Theater & $\begin{array}{l}\text { Microsurgical } \\
\text { labontony }\end{array}$ & $\begin{array}{l}\text { Radiology } \\
\text { CT / MRI }\end{array}$ \\
\hline C & $\begin{array}{l}\text { Radiology } \\
\text { CT / MRI }\end{array}$ & $\begin{array}{l}\text { Surgical trainings } \\
\text { and anatomy }\end{array}$ & OP-Theater & $\begin{array}{c}\text { Microsurgical } \\
\text { labontory }\end{array}$ \\
\hline D & $\begin{array}{l}\text { Microsungical } \\
\text { labontony }\end{array}$ & $\begin{array}{l}\text { Radiology } \\
\text { CT / MRI }\end{array}$ & $\begin{array}{l}\text { Surgical training } \\
\text { and anatormy }\end{array}$ & OP-Thealer \\
\hline
\end{tabular}

Figura 8. Organograma das atividades do MM-6 (cont.)

\begin{tabular}{|c|c|c|c|c|}
\hline \multirow{2}{*}{ Group / Day } & \multicolumn{3}{|c|}{$\begin{array}{l}\text { Praction Work I } \\
\text { 13:30 - 15:00 } \\
\text { July }\end{array}$} & \multirow[b]{2}{*}{$26 / 27 / 29 / 30$} \\
\hline & $5 / 6 / 8 / 9$ & $12 / 13 / 15 / 16$ & $19 / 20 / 22 / 23$ & \\
\hline A & VIDEO & $\begin{array}{c}\text { Doppler } \\
\text { ICU }\end{array}$ & $\begin{array}{c}\text { LAB } \\
\text { Solve o case }\end{array}$ & Neuropsth \\
\hline B & $\begin{array}{l}\text { Dopple } \\
\text { ICU }\end{array}$ & $\begin{array}{c}\text { LAB } \\
\text { Solve a case }\end{array}$ & Neuropath & VIDEO \\
\hline C & $\begin{array}{c}\text { LAB } \\
\text { Solve a case }\end{array}$ & Neuropath. & VIDEO & $\begin{array}{c}\text { Doppler } \\
\text { ICU }\end{array}$ \\
\hline D & Neuropath. & VIDEO & $\begin{array}{l}\text { Doppler } \\
\text { ICU }\end{array}$ & $\begin{array}{c}\text { LAB } \\
\text { Solve o case }\end{array}$ \\
\hline
\end{tabular}

Figura 9. Organograma das atividades do MM-6 (cont.).

da parte anterior do polígono de Willis; anastomose ex tra-intra craniana nas doenças cerebrovasculares oclusivas; cirurgia estereotáxica nos tumores cerebrais.

As quartas-feiras são reservadas para visita a instituições de Berlim e passeios. A programação dos MM é feita de tal forma que a atividade de seus membros se torna agradável, permitindo conhecer pontos turísticos de Berlim e outras cidades próximas como Hamburgo, Hannover, Potsdam, Praga. Já é tradicional a disputa de uma Figura 6. integrantes do MM-6 (1993).partida amistosa de futebol entre as equipes do Klinikum e do MM, (brasileiros). Qualquer que seja o resultado, os alemães oferecem um jantar típico imediatamente após o jogo. Em retribuição, os brasileiros preparam uma feijoada com todos os pertences. O clima de cordialidade tem sido uma constante!

A escolha dos candidatos passou a ser uma tarefa difícil para o Tutor, tendo em vista que a procura aumentava de um para outro MM, a tal ponto que no MM-5, realizado em 1989, a relação foi aproximadamente cinco candidatos para uma vaga, o que fez com que fosse autorizado o aumento do número de vagas para vinte e oito. Os resultados práticos dos Seminários ficaram evidentes. Muitos dos participantes ocupam cargos e postos de destaque no campo da especialidade no Brasil. Os membros dos MM constituem hoje um grupo sui-generis entre os neurocirurgiões brasileiros. Eles se tornaram amigos e a cada encontro que se verifica por ocasião dos Congressos, é grande a alegria com a recordação dos momentos vividos na Alemanha.

O grupo que constituiu o MM-6 decidiu fundar uma Sociedade de Neurocirurgia que congregasse todos os participantes dos diversos MM. No dia 7 de julho de 1993,4 feira, às $21 \mathrm{~h}$ em Berlim, no local denominado "Spree- 7 Athen", na Mommenstrasse, foi fundada a "Sociedade Mário Brock de Neurocirurgia" durante o jantar de boas- $\neg$ vindas. No dia 13 de julho do mesmo mês foi eleita a primeira diretoria, provisória, destinada a estruturar a Sociedade, planejar e realizar a primeira Reunião de caráter científico projetada para a última semana do mês de julho de 1994.

As seguintes cidades brasileiras, por ordem alfabética foram contempladas com a escolha de representantes para esses encontros: Araguaína, Belo Horizonte, Blumenau, Botucatu, Brasília, Campinas, Campos, Canoas, Caruaru, Concórdia, Cuiabá, Curitiba, Fortaleza, Franca, Goiânia, Governador Valadares, Juiz de Fora, Limeira, Londrina, Marília, Natal, Novo Hamburgo, Palmeira das Missões, Pelotas, Petrópolis, Piracicaba, Porto Alegre, Recife, Rio de Janeiro, Ribeirão Preto, Santa Cruz do Sul, Santa Maria, Santa Rosa, São José do Rio Preto, São Leopoldo, São Paulo, Terezina, Uberaba, Viamão, Vitória.

Em nome dos neurocirurgiões participantes dos diversos MM, os autores desejam manifestar seu sincero agradecimento e reconhecimento ao Governo de Berlim, à Sociedade Carlos Duisberg e ao Serviço de Intercâmbio Acadêmico Alemão (D.A.A.D.), pela excelente contribuição. 